\title{
Non poca peritoneale in Italia quanto invece poca peritoneale al Sud
}

Carmine Zoccali

\author{
CNR Centro di Fisiologia Clinica e Dipartimento di Nefro-Urologia \\ Azienda Ospedaliera di Reggio Calabria
}

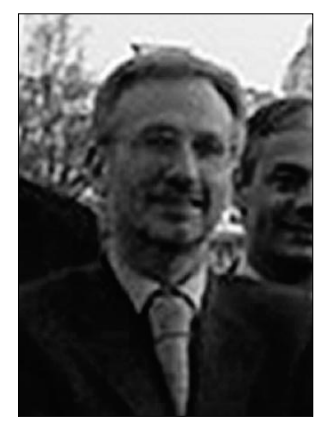

\} 1 \text { problema } dell'uso limitato della dialisi peritoneale in Italia in realtà credo che sia essenzialmente un problema di asimmetria distributiva di questo trattamento nel territorio nazionale. Preliminarmente c'è da fissare uno standard, lo standard di pazienti che possono essere trattati con la dialisi peritoneale ottimizzando $i$ risultati clinici e realizzando ragionevoli risparmi nella spesa sanitaria. Non ci sono certezze al riguardo ma io ritengo che in questa fase storica se su scala nazionale si raggiungesse una media del $15 \%$, quella indicata dal precedente Piano Sanitario Nazionale, probabilmente non saremmo lontani dalla situazione ideale. Le nazioni che applicano la dialisi peritoneale in maniera estensiva, come la Gran Bretagna $(52 \%)$ e il Canada $(37 \%)$, sono anche quelle che hanno avuto una preoccupante limitazione delle risorse destinate alle dialisi e in quei Paesi la qualità dell'assistenza ai nefropatici non può essere certo assunta come modello di riferimento, essendo per molti versi criticabile (1).
Ci sono sostanziali differenze tra la nefrologia del Sud e quella del Nord: in termini di distribuzione territoriale, offerta di servizi, efficienza. Se ci focalizziamo sulla distribuzione territoriale e scegliamo alcune Regioni test, emerge che in Lombardia ci sono globalmente 9 unità operative e CAL per milione di abitanti. Un numero molto vicino lo riscontriamo in Piemonte e in Veneto e mediamente nelle tre regioni si possono contare 12 Centri per milione. Nelle regioni dell'Italia Centro-Nord, Emilia e Toscana, la situazione è simile: in media 11.5 Centri per milione. Nelle 4 regioni più a Sud dell'Italia Campania, Puglia, Calabria e Sicilia la densità è mediamente del $50 \%$ più alta rispetto a quella delle altre Regioni. Quindi non sembra esistere una questione meridionale in nefrologia. I pazienti con uremia terminale hanno apparentemente la stessa opportunità di trattamento indipendentemente da quale sia la loro Regione. Ma per capire bisogna entrare nei dettagli del sistema cioè guardare la gamma dei servizi erogati. La reale differenza è qui. I Centri dialisi italiani, dal Nord al Sud, sono nati come Centri di emodialisi. Tuttavia non tutti i Centri hanno avuto la maturazione organizzativa e professionale necessaria per allargare la gamma dei trattamenti dialitici alla dialisi peritoneale. Me- diamente nelle tre Regioni test del Nord il $15 \%$ dei pazienti vengono trattati con la dialisi peritoneale, nel Centro si scende al $9.5 \%$ e nel Sud si scende ancora al $7.5 \%$, nonostante la buona performance della Calabria e il numero accettabile di trattamenti in Puglia. La dialisi peritoneale viene usata molto meno nel Sud del Paese. Le regioni del Nord sono già attestate e da vari anni, sullo standard auspicabile. È innegabile che la presenza della dialisi privata è quello che fa la differenza. La dialisi peritoneale non è praticata virtualmente da nessuna struttura privata e $\mathrm{i}$ trattamenti sostitutivi sono gestiti in larga parte dalla Sanità privata nelle tre regioni italiane che praticano meno di tutte la dialisi peritoneale: Campania, Sicilia, Lazio. La causa di questo secondo me non è tanto il fatto che la dialisi peritoneale non possa essere remunerativa quanto il fatto che questo tipo di dialisi richiede un assetto organizzativo più complesso e che creare questo assetto complesso nel quale, per esempio, un certo numero di letti di degenza e monitors siano riservati ai pazienti con complicanze cliniche richiederebbe investimenti infrastrutturali, in risorse umane e organizzative che la Sanità privata non ha nessuna ragione per fare perché, comunque, in termini finanziari il ritorno economico globale rimarrebbe 
inferiore a quello dell'emodialisi. Ma qui non si tratta di demonizzare la Sanità privata che, ovviamente, produce e può produrre come quella pubblica un'assistenza di qualità. Il problema è che la Sanità privata risponde esclusivamente a stimoli di natura finanziaria e alle richieste del mercato mentre la Sanità pubblica, avendo un'altra missione, è più aperta a sperimentare. Almeno finora, la protezione del sistema sanitario nazionale ha consentito ai Centri pubblici italiani di essere tra i più innovativi in campo internazionale nella sperimentazione sulla dialisi peritoneale e di sviluppare con successo questa forma di trattamento che alla fine risulta vantaggiosa anche dal punto vista economico. Un'espansione della dialisi peritoneale nel Sud potrà verificarsi solo se si introdurranno stimoli finanziari e una regolamentazione adeguata. È da escludere infatti, anche per l'assetto politico che il nostro Paese molto probabilmente avrà nei prossimi anni, che la Sanità privata deciderà di investire su un settore il cui ritorno finanziario è quanto meno incerto. Senza la spinta specifica di porre come obiettivo lo sviluppo della dialisi peritoneale come un obiettivo ben identificato nell'organizzazione sanitaria e senza introdurre coerenti incentivi finanziari è illusorio attendersi che qualcosa possa cambiare.

carmine.zoccali@tin.it

\section{BIBLIOGRAFIA}

1.

Horl WH, de Alvaro F, Williams PF. Healthcare systems and end-stage renal disease (ESRD) therapies-an international review: access to ESRD treatments. Nephrol Dial Transplant 1999;14 (suppl.): S10-5 (Review). 